\title{
Vascular Steal Explains Early Paradoxical Blood Oxygen Level-Dependent Cerebrovascular Response in Brain Regions with Delayed Arterial Transit Times
}

\author{
Julien Poublanc Jay Show Han Daniel Michael Mandell John Conklin \\ Jeffrey Alan Stainsby Joseph Arnold Fisher David John Mikulis \\ Adrian Philip Crawley \\ Department of Medical Imaging, Toronto Western Hospital, Toronto, Ont., Canada
}

\section{Key Words}

Steal phenomenon · Time delay · Blood oxygen level-dependent magnetic resonance imaging $\cdot$ Cerebrovascular reactivity $\cdot$ Dynamic contrast imaging

\section{Abstract}

Introduction: Blood oxygen level-dependent (BOLD) magnetic resonance imaging (MRI) during manipulation of inhaled carbon dioxide $\left(\mathrm{CO}_{2}\right)$ can be used to measure cerebrovascular reactivity (CVR) and map regions of exhausted cerebrovascular reserve. These regions exhibit a reduced or negative $\mathrm{BOLD}$ response to inhaled $\mathrm{CO}_{2}$. In this study, we sought to clarify the mechanism behind the negative BOLD response by investigating its time delay (TD). Dynamic susceptibility contrast (DSC) MRI with the injection of a contrast agent was used as the gold standard in order to provide measurement of the blood arrival time to which CVR TD could be compared. We hypothesize that if negative BOLD responses are the result of a steal phenomenon, they should be synchronized with positive BOLD responses from healthy brain tissue, even though the blood arrival time would be delayed. Methods: On a 3-tesla MRI system, BOLD CVR and DSC images were collected in a group of 19 patients with steno-occlusive cerebrovascular disease. For each patient, we generated a CVR magnitude map by regressing the BOLD signal with the end-tidal partial pressure of $\mathrm{CO}_{2}\left(\mathrm{P}_{\mathrm{ET}} \mathrm{CO}_{2}\right)$, and a CVR TD map by extracting the time of maximum cross-correlation between the BOLD signal and $\mathrm{PET}_{\mathrm{ET}} \mathrm{CO}_{2}$. In addition, a blood arrival time map was generated by fitting the DSC signal with a gamma variate function. ROI masks corresponding to varying degrees of reactivity were constructed. Within these masks, the mean CVR magnitude, CVR TD and DSC blood arrival time were extracted and averaged over the 19 patients. CVR magnitude and CVR TD were then plotted against DSC blood arrival time. Results: The results show that CVR magnitude is highly correlated to 
Poublanc et al.: Vascular Steal Explains Early Paradoxical Blood Oxygen Level-Dependent Cerebrovascular Response in Brain Regions with Delayed Arterial Transit Times

DSC blood arrival time. As expected, the most compromised tissues with the longest blood arrival time have the lowest (most negative) CVR magnitude. However, CVR TD shows a noncontinuous relationship with DSC blood arrival time. CVR TD is well correlated to DSC blood arrival time $(p<0.0001)$ for tissue of positive reactivity, but fails to maintain this trend for tissue of negative reactivity. Regions with negative reactivity have similar CVR TD than healthy regions. Conclusion: These results support the hypothesis that negative reactivity is the result of a steal phenomenon, lowering the BOLD signal as soon as healthier parts of the brain start to react and augment their blood flow. BOLD CVR MRI is capable of identifying this steal distribution, which has particular diagnostic significance as it represents an actual reduction in flow to already compromised tissue.

Copyright $\odot 2013$ S. Karger AG, Basel

\section{Introduction}

Blood oxygen level-dependent (BOLD) magnetic resonance imaging (MRI) can be used to measure cerebrovascular reactivity (CVR) to vasodilators such as carbon dioxide $\left(\mathrm{CO}_{2}\right)$. In healthy brain tissue, increased end-tidal partial pressure of $\mathrm{CO}_{2}\left(\mathrm{P}_{\mathrm{ET}} \mathrm{CO}_{2}\right)$ causes arteriolar dilation, producing an increase in blood flow and an increased BOLD MRI signal. In patients with steno-occlusive disease, it is expected that compromised tissue will become chronically vasodilated and may exhibit an inability to further augment blood flow, which would lead to an absence of any BOLD response to a $\mathrm{CO}_{2}$ stimulus [1]. In fact, a significant decrease in BOLD signal is often seen in tissue that is presumed to be severely compromised [2]. This decrease in BOLD signal must correspond to an increase in the amount of deoxyhemoglobin within the voxel, which might be explained by either of two physiological models.

We hypothesize that either a somewhat time-delayed volume increase occurs due to local vasodilation but the overall resistance including proximal stenoses does not allow for any significant increase in blood flow (model A) or other healthier tissue vasodilates before the compromised tissue under consideration and the immediate pressure drop causes an actual decrease in flow (model B). This second mechanism, named vascular steal phenomenon, has previously been described [3]. It occurs in brain regions with increased proximal resistance such as an arterial stenosis. In this case, the arterioles experience a lowered perfusion pressure and respond over time by a chronic compensatory vasodilation. This is effective in maintaining resting blood flow until maximal vasodilation is reached. At this point, if a vasodilator stimulus is delivered to the brain, the compromised tissue is unable to reduce its resistance, and blood flow is diverted to surrounding healthy tissues with preserved vasodilatory capacity.

In this retrospective study, we attempt to distinguish those two models ( $\mathrm{A}$ and $\mathrm{B}$ ) on the basis of time delay (TD) measurements. We compare BOLD CVR scans with dynamic susceptibility contrast (DSC) imaging in a group of patients with steno-occlusive disease. The DSC technique uses an intravenous injection of a paramagnetic contrast agent (gadolinium) in order to induce a large MR signal drop via an intravascular T2* decrease. The relative arrival time of the gadolinium contrast in the capillary beds can be measured fairly accurately and provides a surrogate estimate for the relative arrival times of the arterial $\mathrm{CO}_{2}$ bolus that is created by modulation of the inhaled gas partial pressure of $\mathrm{CO}_{2}\left(\mathrm{PCO}_{2}\right)$. The true $\mathrm{CVR}$ response must occur after the arrival of the $\mathrm{CO}_{2}$ bolus. However, any change in pressure across the proximal resistance will occur as soon as the arterioles in the healthier tissue begin to dilate. Therefore, if hypothesis B is true, a negative CVR response due to vascular steal should occur in synchrony with the positive response of the healthier tissue causing the steal. 


\section{Cerebrovascular \\ Diseases}

Table 1. Patient characteristics \begin{tabular}{l|l}
\hline Cerebrovasc Dis Extra 2013;3:55-64 \\
\hline DOI: 10.1159/000348841 & $\begin{array}{l}\text { C 2013 S. Karger AG, Basel } \\
\text { www.karger.com/cee }\end{array}$ \\
\hline Poublanc et al.: Vascular Steal Explains Early Paradoxical Blood Oxygen Level-Dependent
\end{tabular} Cerebrovascular Response in Brain Regions with Delayed Arterial Transit Times
Patient No./ Pathology

sex/age

$1 / \mathrm{M} / 15$

2/M/15

$3 / \mathrm{F} / 17$

$4 / \mathrm{F} / 18$

5/M26

$6 / \mathrm{M} / 36$

$7 / \mathrm{M} / 45$

$8 / \mathrm{M} / 46$

9/F/48

$10 / \mathrm{M} / 56$

$11 / \mathrm{F} / 60$

$12 / \mathrm{M} / 60$

13/M/69

14/M/71

$15 / \mathrm{F} / 74$

$16 / \mathrm{M} / 74$

$17 / \mathrm{M} / 75$

$18 / \mathrm{F} / 83$

$19 / \mathrm{M} / 60$

ICA = Internal carotid artery; MCA = middle cerebral artery.

\section{Methods}

\section{Study Population}

The study protocol was approved by the University Health Network Research Ethics Board. A total of 19 patients (table 1) with steno-occlusive cerebrovascular disease, referred from the neurology and neurosurgery services at the Toronto Western Hospital, were recruited into this study. All patients provided written informed consent.

\section{Imaging Protocol}

All patients were imaged on a 3-tesla MRI system (GE Medical Systems, Milwaukee, Wisc., USA), using an 8-channel head coil.

\section{BOLD CVR}

CVR images were acquired using a BOLD MR pulse sequence with the following parameters: $\mathrm{TR}=2,000 \mathrm{~ms}, \mathrm{TE}=35 \mathrm{~ms}$, flip angle $=85^{\circ}, 28$ slices, slice thickness $=4.5 \mathrm{~mm}, 64 \times 64$ matrix size, field of view $=200 \mathrm{~mm}$, and either a spiral or echo planar imaging readout.

\section{CVR Equipment and Protocol}

Gas delivery to the patient was achieved using a custom-built automated gas blender and breathing circuit (RespirAct; Thornhill Research Inc., Toronto, Ont., Canada). This apparatus described by Slessarev et al. [4] enables the prospective control of the patient's $\mathrm{P}_{\mathrm{ET}} \mathrm{CO}_{2}$ and end-tidal pressure $\mathrm{O}_{2}\left(\mathrm{P}_{\mathrm{ET}} \mathrm{O}_{2}\right)$ independently of each other and of minute ventilation $[5,6]$.

During BOLD MRI acquisition, patients were induced with alternating normocapnic $(\mathrm{N})$ and hypercapnic $(\mathrm{H})$ states during iso-oxia $\left(\mathrm{P}_{\mathrm{ET}} \mathrm{O}_{2}=100 \mathrm{~mm} \mathrm{Hg}\right)$. Normocapnic and hypercapnic states refer to $\mathrm{P}_{\mathrm{ET}} \mathrm{CO}_{2}=40 \mathrm{~mm} \mathrm{Hg}$ and $\mathrm{P}_{\mathrm{ET}} \mathrm{CO}_{2}=50 \mathrm{~mm} \mathrm{Hg}$, respectively. The timing sequence was as follows: $\mathrm{N} / \mathrm{H} / \mathrm{N} / \mathrm{H} / \mathrm{N}=45 / 45 / 90 / 150 / 180 \mathrm{~s}$. 
Even though the above describes the most current material used in our institution, a more basic rebreathing system (where $\mathrm{P}_{\mathrm{ET}} \mathrm{O}_{2}$ is not controlled) was used in 13 patients. In these cases, normocapnic ( $40 \mathrm{~mm} \mathrm{Hg}$ ) and hypercapnic (50 $\mathrm{mm} \mathrm{Hg}$ ) states were alternating periodically for about $1 \mathrm{~min}$ [see 7 for details].

Anatomical Scan

For coregistration purposes, T1-weighted images were acquired using an inversion recovery-prepared spoiled gradient-recalled sequence with the following parameters: flip angle $=20^{\circ}, \mathrm{TI} / \mathrm{TE} / \mathrm{TR}=300 / 5 / 11.5 \mathrm{~ms}, 60$ slices of $2.2 \mathrm{~mm}$ thickness and a $256 \times 256$ matrix size .

DSC Imaging Scan

T2*-weighted dynamic images were acquired using gradient-echo, single-shot echo planar imaging $\left(\mathrm{TR}=1,735 \mathrm{~ms}\right.$, TE $=31.5 \mathrm{~ms}$, flip angle $=90^{\circ}, 128 \times 128$ acquisition matrix, $20 \times 20 \mathrm{~cm}$ field of view, 18 slices of $5 \mathrm{~mm}$ thickness and $2 \mathrm{~mm}$ gap) during a 15-ml intravenous injection of $7.5 \mathrm{mmol}$ of gadodiamide (Omniscan) at a flow rate of $5 \mathrm{ml} / \mathrm{s}$, immediately followed by a $30-\mathrm{ml}$ saline flush.

\section{Data Processing}

End-Tidal Partial Pressure of $\mathrm{CO}_{2}$

$\mathrm{P}_{\mathrm{ET}} \mathrm{CO}_{2}$ was obtained by selecting the expiration peaks of the $\mathrm{PCO}_{2}$ curve and used as a reference waveform in the cross-correlation analysis.

MRI Data

All data were transferred to an independent workstation for further analysis. For each raw data set (CVR and DSC), slice-time correction, followed by volume re-registration for motion correction during scanning, was applied using the AFNI software [8]. Next, CVR and DSC volumes were realigned onto the anatomical image to correct for possible changes in the patient's head position between scans.

CVR Magnitude or Reactivity Map

To account for the unknown delay between the $\mathrm{P}_{\mathrm{ET}} \mathrm{CO}_{2}$ waveform and the actual arterial $\mathrm{PCO}_{2}$ in the brain, the $\mathrm{P}_{\mathrm{ET}} \mathrm{CO}_{2}$ waveform was first time-shifted to correspond to the maximum cross-correlation with the BOLD signal averaged over the brain. The BOLD time series at each voxel was then normalized and regressed against the $\mathrm{P}_{\mathrm{ET}} \mathrm{CO}_{2}$ waveform. The slope of this regression is the magnitude of the CVR signal and is expressed as a percentage change in BOLD signal per mm $\mathrm{Hg}(\% / \mathrm{mm} \mathrm{Hg})$ in $\mathrm{P}_{\mathrm{ET}} \mathrm{CO}_{2}$. The final CVR magnitude map (M; fig. 1a) is displayed with a graded color scale, and can be thresholded with the correlation coefficient resulting from the cross-correlation between the BOLD signal and $\mathrm{P}_{\mathrm{ET}} \mathrm{CO}_{2}$.

DSC Blood Arrival Time Map

AFNI was used to perform a nonlinear fitting procedure of the DSC time curve by a gamma variate of the form: $S=\left(t-t_{0}\right)^{a} \times \exp \left(-\left(t-t_{0}\right) / b\right), t_{0}$ being the start of the signal or blood arrival time (BAT). In this way, BAT was calculated for each voxel, and the resulting map (fig. 1b) was resampled to match the CVR grid.

CVR TD Map

An AFNI program called the Hilbert Delay $[9,10]$ was used to calculate the TD maps (fig. 1c). This program estimates the time at which the cross-correlation between the $\mathrm{P}_{\mathrm{ET}} \mathrm{CO}_{2}$ and the BOLD signal time course is a maximum. The advantage of this method is its ability to generate 


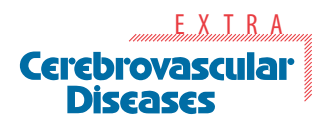

\begin{tabular}{l|l}
\hline \multicolumn{2}{l}{ Cerebrovasc Dis Extra 2013;3:55-64 } \\
\hline DOI: $10.1159 / 000348841$ & $\begin{array}{l}\text { C } 2013 \text { S. Karger AG, Basel } \\
\text { www.karger.com/cee }\end{array}$ \\
\hline
\end{tabular}

Poublanc et al.: Vascular Steal Explains Early Paradoxical Blood Oxygen Level-Dependent Cerebrovascular Response in Brain Regions with Delayed Arterial Transit Times

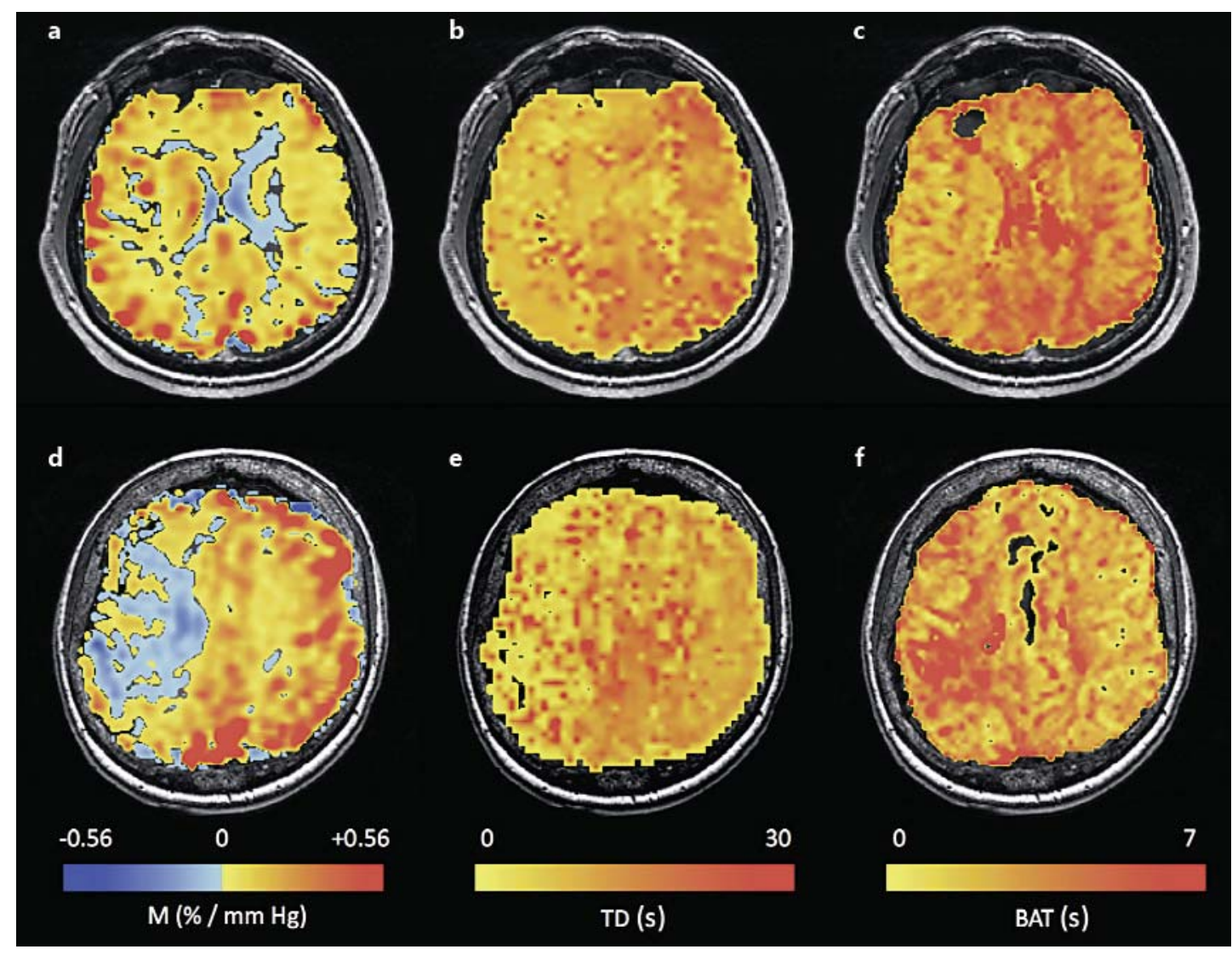

Fig. 1. CVR magnitude or $M(\mathbf{a}, \mathbf{d})$, TD (b, e) and BAT (c, $\mathbf{f})$ maps for 2 patients. The first row (a-c) represents a patient with mainly positive reactivity, whereas the second row $(\mathbf{d}-\mathbf{f})$ represents a patient with an extended area of negative reactivity.

TDs at a finer resolution than TR without having to entirely interpolate the signal and reference waveforms. It takes advantage of the fact that the cross-correlation is maximum as its Hilbert transform crosses zero. The zero crossing time point between two TRs is easily found with one linear interpolation. In this way, TD, along with a corresponding correlation value, was calculated on a voxel-by-voxel basis. Additionally, TD in voxels of negative reactivity, determined from the initial $\mathrm{M}$ map $(\mathrm{M}<0)$, was calculated using an inverted $\mathrm{P}_{\mathrm{ET}} \mathrm{CO}_{2}$ waveform. The TD map obtained is relative to the $\mathrm{P}_{\mathrm{ET}} \mathrm{CO}_{2}$. Note that on a per patient basis, the position of the $\mathrm{P}_{\mathrm{ET}} \mathrm{CO}_{2}$ is not synchronized with the healthy brain but with the average brain signal. We later adjusted each patient's map to a common reference in order to group all 19 patients together.

Also, it is important to realize that TD as defined above is very different than BAT. Due to the nature of the cross-correlation, it is a more general time combining a BAT as well as a response time. Response time could be defined as the time for the signal to go from baseline to maximum reactivity. TD differences between healthy and diseased tissue can be as long as half a minute in some patients, whereas corresponding BAT differences are on the order of a few seconds.

\section{Data Analysis}

For each patient, whole-brain CVR data were separated into multiple intervals or bins of M spanning $0.05 \% / \mathrm{mm} \mathrm{Hg}$ each and ranged from -0.20 to $0.60 \% / \mathrm{mm} \mathrm{Hg}$. This method was used to construct 16 corresponding ROI masks. Additionally, as the program Hilbert Delay 


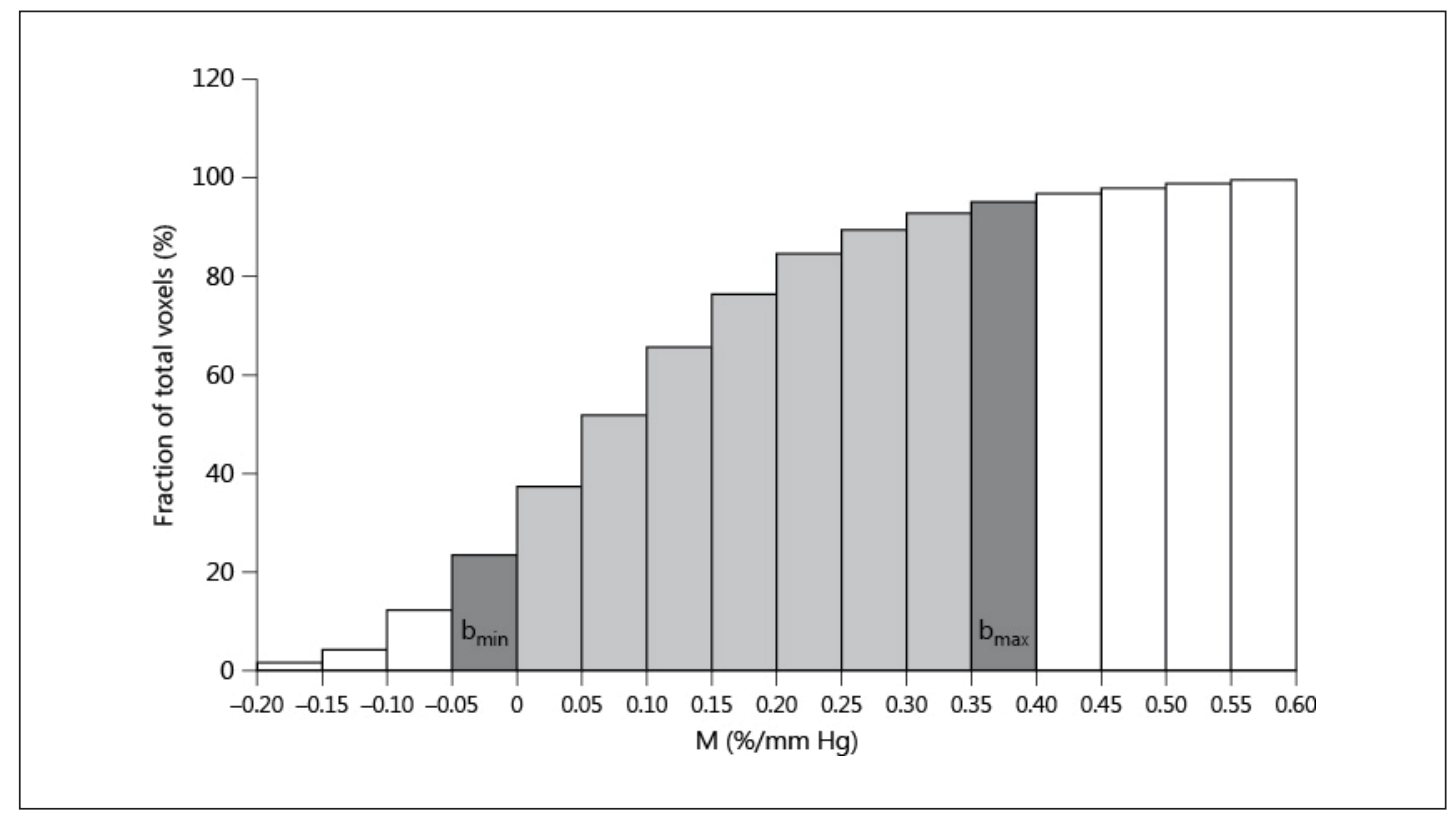

Fig. 2. Cumulative histogram of reactivity $(M)$ for 1 patient. It is used to define the patient range of reactivity. The highest $5 \%$ and the lowest $5 \%$ of all $M$ values are excluded from the analysis (white bins). In dark and light grey are all the included bins for this patient. In dark grey are the minimum and maximum reactivity bins ( $b_{\min }$ and $b_{\max }$, respectively).

can potentially yield extremely long delays (physiologically impossible) for noisy time series, those masks were further restricted by excluding any voxel of TD greater than $45 \mathrm{~s}$.

For each patient, the number of voxels contained in each of the 16 ROI masks was used to construct the cumulative histogram (fig. 2). To reduce the influence of outliers caused by large veins (dural sinuses) or movement artifacts, we removed the highest $5 \%$ and the lowest $5 \%$ of all $M$ values. As shown in figure 2 , this defines the patient's range of reactivity, with $b_{\text {min }}$ and $b_{\max }$ being the minimum and maximum bin included.

In each patient, average M, TD and BAT were calculated for each of the corresponding ROI masks included in the reactivity range. Since TD and BAT are not absolute parameters, a common reference between all patients must be defined. We assume that healthy tissue with maximum reactivity should have the earliest TD and BAT. Consequently, for each patient separately, we used the ROI mask of $b_{\max }$ as the zero reference and shifted all TD and BAT accordingly. It was then possible to average $\mathrm{M}, \mathrm{TD}$ and BAT across patients for each separate bin. Note that the number of patients contributing to different ROI masks (constructed from reactivity intervals) can vary as the individual range of reactivity varies from patient to patient. Final averaged $\mathrm{M}$ and TD were plotted against averaged BAT (fig. 2).

\section{Results}

Figure 1 shows the CVR M, TD and BAT for 2 patients. The first patient shows a good correspondence between positive diminished reactivity (fig. 1a) and prolonged TD (fig. 1b) and BAT (fig. 1c). In the second patient, the region of negative reactivity (fig. $1 \mathrm{~d}$ ) has a prolonged BAT (fig. 1f) but a short TD (fig. 1e). 
Poublanc et al.: Vascular Steal Explains Early Paradoxical Blood Oxygen Level-Dependent Cerebrovascular Response in Brain Regions with Delayed Arterial Transit Times

Fig. 3. CVR magnitude (M) plotted against DSC BAT (a) as well as CVR TD plotted against DSC BAT (b) for all patients. Positive and negative reactivity is represented, respectively, in black and grey. a The number of patients represented by each data point is shown in grey.

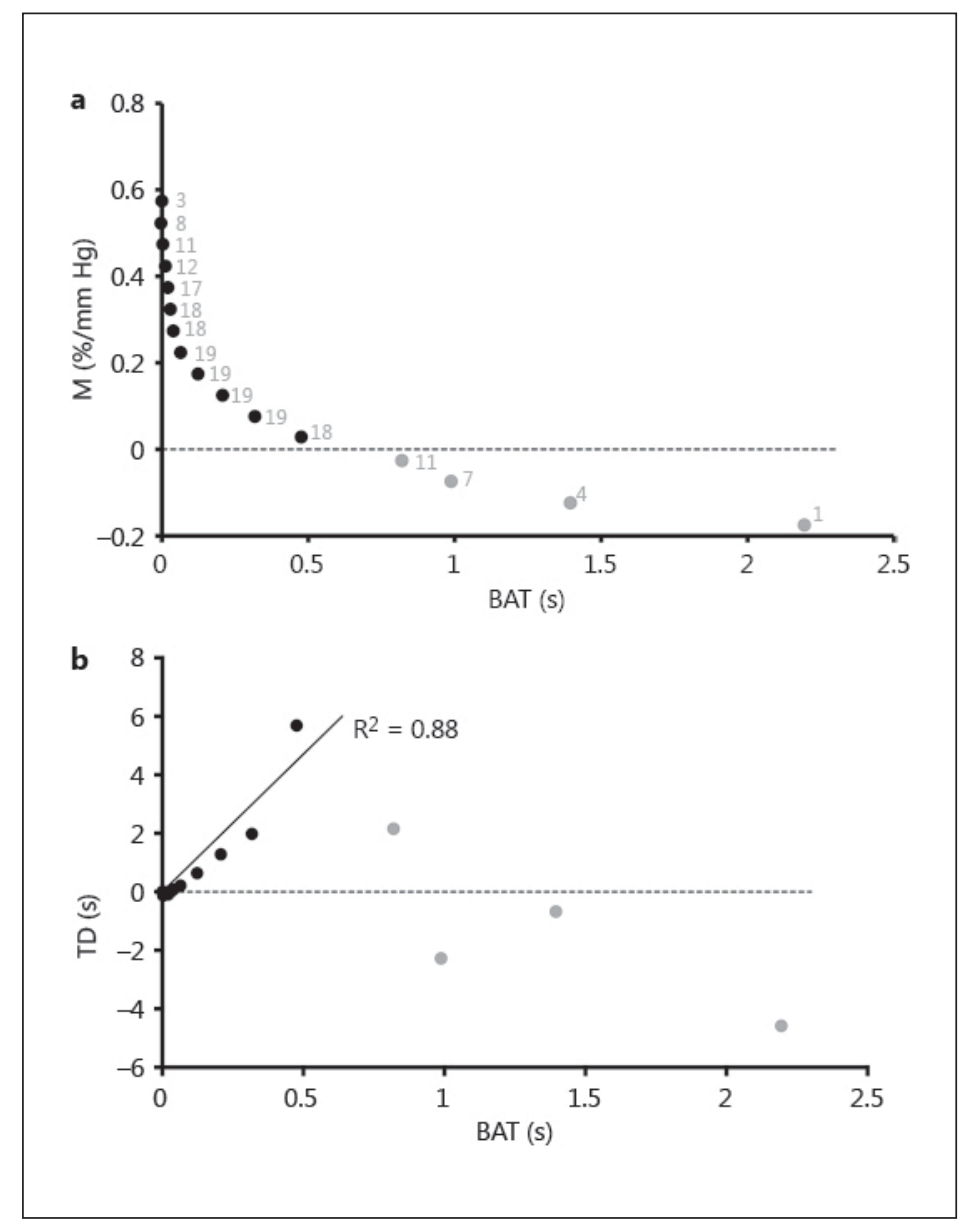

Figure 3a shows CVR M plotted against DSC BAT. Black and grey data points represent the positive and negative reactivity data, respectively. Each of the data points corresponds to one of the bins. Next to each data point is the number of patients contributing to the average $\mathrm{M}$ and BAT (grey text). This graph clearly shows that M and BAT are related.

Figure $3 \mathrm{~b}$ shows CVR TD plotted against DSC BAT. Regression was used to analyze these data, with the constraint of BAT and TD being simultaneously null in healthy tissue. If all black and grey data points are taken into account, TD is not correlated to BAT $\left(n=16, R^{2}=0.09\right.$, $p=0.27$ ). However, if only data from regions of positive reactivity (M) are considered (black data points), TD is well correlated to BAT ( $\left.\mathrm{n}=12, \mathrm{R}^{2}=0.88, \mathrm{p}<0.0001\right)$. As expected (see Methods section) TD is much longer than BAT. Note that there is a 10 -fold difference between the two parameters.

\section{Discussion}

The conventional parameter extracted from a BOLD CVR experiment is the magnitude of the BOLD response expressed as a percent BOLD signal increase per $\mathrm{mm} \mathrm{Hg} \mathrm{P}_{\mathrm{ET}} \mathrm{CO}_{2}$. Figure 3a shows that this parameter, $\mathrm{M}$, is well correlated to the BAT parameter estimated by the DSC technique. This implies that the two techniques detect vascular impairment in similar brain regions. 
Poublanc et al.: Vascular Steal Explains Early Paradoxical Blood Oxygen Level-Dependent Cerebrovascular Response in Brain Regions with Delayed Arterial Transit Times

However, we observed that healthy tissue with no BAT delay (BAT $\approx 0$ ) has a surprisingly wide range of reactivity $(\mathrm{M} \approx 0.4-0.6 \% / \mathrm{mm} \mathrm{Hg}$ ) illustrated by the steep descending portion of the curve in figure $3 \mathrm{a}$. This may reflect partial volume effects in voxels containing large draining veins. As those voxels have a greater blood volume than voxels containing only tissue, deoxyhemoglobin content and subsequently BOLD signal will be much larger. The steepness of the curve around zero BAT would decrease if large draining veins were masked out. This effect was reduced by defining the patient reactivity range illustrated in figure 2 .

In contrast to the continuous relationship between $M$ and BAT seen in figure 3a, TD plotted against BAT (fig. $3 \mathrm{~b}$ ) shows that voxels with negative reactivity (grey data points) behave very differently from voxels with positive reactivity (black data points). From figure $3 \mathrm{a}$, we know that the voxels with negative reactivity correspond to the most impaired vasculature because the reference technique (DSC) shows that they experience the longest BAT. However, these voxels (grey data points) are not detected as impaired vasculature by CVR TD. Indeed, TD increases linearly with BAT for positive M, but this trend fails spectacularly when $\mathrm{M}$ becomes negative. The grey data points have the lowest TD, which conflicts with any expectation that increasing TD may reflect increased severity of disease, and this would be consistent with hypothesis A. We interpret this result as evidence for hypothesis B, i.e. that negative reactivity is the result of a steal phenomenon, which lowers the BOLD signal in severely diseased tissue as soon as healthier parts of the brain start to react and augment their blood flow.

Our observation that negative reactivity is secondary to a steal phenomenon is supported by previous work in subjects with high-grade vascular stenosis [11]. In addition, arterial spin labeling, which provides a more direct measure of blood flow, indicates a reduced blood flow in tissue that shows a reduced BOLD CVR magnitude [12].

The vasculature in regions with negative 'reactivity' is not reacting to a local increase in $\mathrm{P}_{\mathrm{ET}} \mathrm{CO}_{2}$. Rather, the reduction in blood flow is driven passively by the dilation of vessels in the surrounding healthier tissue. The DSC data demonstrate that the gadolinium bolus and equivalently the $\mathrm{CO}_{2}$ bolus usually arrive at the more compromised tissue slightly later than at the surrounding healthier tissue. As the healthier tissue begins to vasodilate, a greater pressure drop across the stenosed arteries will reduce the perfusion pressure simultaneously at all downstream arterioles. We expect the BOLD signal from severely compromised tissue that is susceptible to a steal effect to start to change in exact antiphase to the signal from the normally reacting healthier tissue.

However, as observed in figure $3 \mathrm{~b}$, three of the grey data points have a shorter TD than the reference TD (TD $=0$ for $\mathrm{M}_{\max }$ ) in healthy tissue. In order to understand this paradoxical result, it is important to realize that the negative BOLD signal is not an exact reflection (about the time axis) of the positive reactivity found in healthy vasculature (fig. 4). Visual examination of a negative BOLD time series during the hypercapnic stage shows that in some cases a slow increase in signal can follow the initial drop (fig. 4, grey line). We conclude that the negative BOLD signal is in general a combination of a steal phenomenon with a small delayed local (positive) reactivity. These two effects introduce an asymmetry in the signal shape and shift the TD calculation toward the initial drop, where the steal phenomenon dominates. This artifact explains how a few (grey) data points can have negative TD.

In this study, the TD parameter was only used to confirm the existence of cerebral steal physiology but could provide useful diagnostic information regarding the nature of the hemodynamic impairment. In particular, TD could be used to locate collateral circulation, which is very important for flow compensation in patients with intracranial stenosis [13]. Also, it should be noted that a previous study [14] using transcranial Doppler found that the delay between the decrease in $\mathrm{P}_{\mathrm{ET}} \mathrm{CO}_{2}$ and the subsequent decrease in cerebral blood flow velocity 


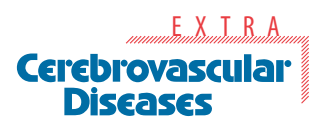

Fig. 4. The raw BOLD signal for 2 voxels from the same patient is shown. One voxel demonstrates positive reactivity to $\mathrm{CO}_{2}$ (black line) while the other demonstrates negative reactivity (grey line). The $\mathrm{P}_{\mathrm{ET}} \mathrm{CO}_{2}$ (dashed line) varies between approximately 40 and 50 $\mathrm{mm} \mathrm{Hg}$. The grey signal shows an initial drop as soon as $\mathrm{PCO}_{2}$ is elevated. However, this is followed by a signal increase during the high $\mathrm{PCO}_{2}$ plateau, which we interpret as a combination of some residual component of true reactivity (vasodilation of vascular beds within this voxel) coupled with steal phenomenon as described in the text.

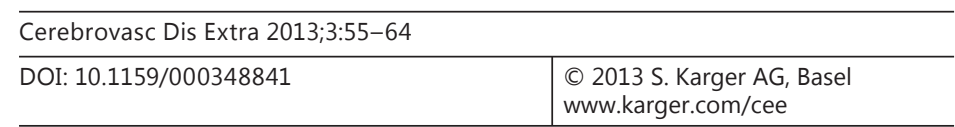

Poublanc et al.: Vascular Steal Explains Early Paradoxical Blood Oxygen Level-Dependent Cerebrovascular Response in Brain Regions with Delayed Arterial Transit Times

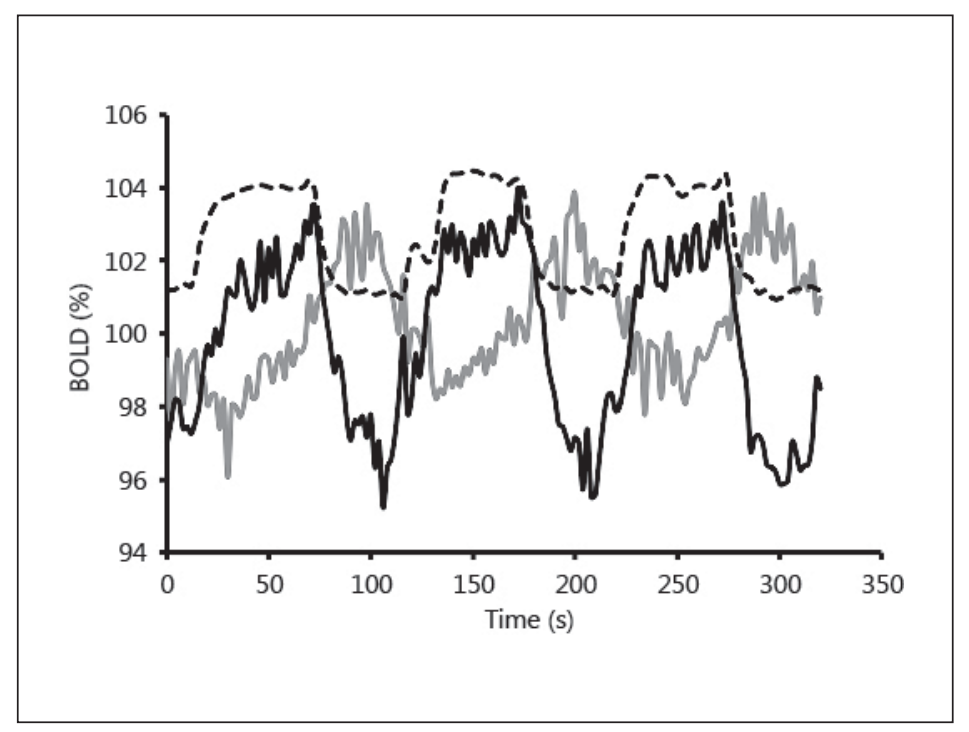

correlated with cerebral hemodynamic compromise. However, extracting time information from a low signal-to-noise time series such as BOLD CVR is challenging especially in impaired vasculature with reactivity approaching zero. Furthermore, we now know that the timing of a negative BOLD signal does not reflect local response time to the vasodilatory stimulus. Moreover, this parameter can normally only be interpreted relative to surrounding areas (in this work, TD was expressed relative to the TD for healthy tissue).

In contrast, the CVR magnitude does not have these disadvantages. First, M is stable even in low reactivity regions, since it is simply a measure of the amplitude of the signal. Second, $\mathrm{M}$ is an absolute measure at each voxel, and is scaled per $\mathrm{mm} \mathrm{Hg}$, making the maps comparable between patients.

\section{Conclusion}

Despite their fundamental difference, BOLD CVR magnitude maps and DSC BAT maps detected vascular impairment in similar locations. Comparison of CVR TDs with DSC BAT confirmed the existence of a steal phenomenon which explains the apparently paradoxical nature of a negative BOLD response to hypercapnia in severely compromised tissue.

A negative BOLD response might be considered particularly diagnostically significant, since it does represent an actual reduction in flow to already compromised tissue, at least immediately following a global hypercapnia challenge. The point at which steal occurs depends upon the resistance of the stenosed proximal vessels and the resistance of the tissue that demonstrates the flow reduction. In a sense, the global stimulus mimics an ischemic event for the tissue at risk.

BOLD CVR MRI is capable of identifying this steal distribution, which can be viewed as preischemic risk imaging. The clinical relevance of identifying this tissue at risk is the severalfold higher incidence of ischemic injury in patients with this physiology [15-18]. 


\section{Cerebrovascular Diseases}

\begin{tabular}{l|l}
\hline \multicolumn{2}{l}{ Cerebrovasc Dis Extra 2013;3:55-64 } \\
\hline DOI: $10.1159 / 000348841$ & $\begin{array}{l}\text { ( ) 2013 S. Karger AG, Basel } \\
\text { www.karger.com/cee }\end{array}$ \\
\hline
\end{tabular}

Poublanc et al.: Vascular Steal Explains Early Paradoxical Blood Oxygen Level-Dependent Cerebrovascular Response in Brain Regions with Delayed Arterial Transit Times

\section{Acknowledgements}

We thank the Toronto Western Hospital MRI technologists Eugen Hlasny, David Johnstone, Keith Ta and Hien Tran for their contributions to the data acquisition. This work was supported by the Ontario Research Fund.

\section{Disclosure Statement}

Two of the study authors (J.A.F. and D.J.M.) contributed to the development of RespirAct $^{\mathrm{TM}}$. These authors stand to gain financially if the device is successfully commercialized by Thornhill Research Inc., a University of Toronto/University Health Network-related company.

\section{References}

1 Mikulis DJ, Krolczyk G, Desal H, Logan W, Deveber G, Dirks P, Tymianski M, Crawley A, Vesely A, Kassner A, Preiss D, Somogyi R, Fisher JA: Preoperative and postoperative mapping of cerebrovascular reactivity in moyamoya disease by using blood oxygen level-dependent magnetic resonance imaging. J Neurosurg 2005; 103:347-355.

-2 Ziyeh S, Rick J, Reinhard M, Hetzel A, Mader I, Speck O: Blood oxygen level-dependent MRI of cerebral $\mathrm{CO}_{2}$ reactivity in severe carotid stenosis and occlusion. Stroke 2005;36:751-756.

- 3 Akiyama T, Ohira T, Kato T, Toda Y, Orii M, Hiraga K, Fukunaga A, Kobayashi M, Onozuka S, Kawase T: Motorrelated intracortical steal phenomenon detected by multichannel functional near-infrared spectroscopy imaging. Cerebrovasc Dis 2005;20:337-346.

4 Slessarev M, Han J, Mardimae A, Prisman E, Preiss D, Volgyesi G, Ansel C, Duffin J, Fisher JA: Prospective targeting and control of end-tidal $\mathrm{CO}_{2}$ and $\mathrm{O}_{2}$ concentrations. J Physiol (Lond) 2007;581:1207-1219.

-5 Somogyi RB, Vesely AE, Preiss D, Prisman E, Volgyesi G, Azami T, Iscoe S, Fisher JA, Sasano H: Precise control of end-tidal carbon dioxide levels using sequential rebreathing circuits. Anaesth Intensive Care 2005;33:726732.

6 Prisman E, Slessarev M, Han J, Poublanc J, Mardimae A, Crawley A, Fisher J, Mikulis D: Comparison of the effects of independently-controlled end-tidal $\mathrm{PCO}_{2}$ and $\mathrm{PO}_{2}$ on blood oxygen level-dependent (BOLD) MRI. J Magn Reson Imaging 2008;27:185-191.

7 Vesely A, Sasano H, Volgyesi G, Somogyi R, Tesler J, Fedorko L, Grynspan J, Crawley A, Fisher JA, Mikulis D: MRI mapping of cerebrovascular reactivity using square wave changes in end-tidal $\mathrm{PCO}_{2}$. Magn Reson Med 2001; 45:1011-1013.

-8 Cox RW: AFNI: software for analysis and visualization of functional magnetic resonance neuroimages. Comput Biomed Res 1996;29:162-173.

-9 Saad ZS, Ropella KM, Cox RW, DeYoe EA: Analysis and use of FMRI response delays. Hum Brain Mapp 2001; 13:74-93.

10 Saad ZS, DeYoe EA, Ropella KM: Estimation of FMRI response delays. Neuroimage 2003;18:494-504.

-11 Furst H, Hartl WH, Janssen I: Patterns of cerebrovascular reactivity in patients with unilateral asymptomatic carotid artery stenosis. Stroke 1994;25:1193-1200.

-12 Mandell DM, Han JS, Poublanc J, Crawley AP, Stainsby JA, Fisher JA, Mikulis DJ: Mapping cerebrovascular reactivity using blood oxygen level-dependent MRI in patients with arterial steno-occlusive disease: comparison with arterial spin labeling MRI. Stroke 2008;39:2021-2028.

13 Lau AY, Wong EH, Wong A, Mok VC, Leung TW, Wong KS: Significance of good collateral compensation in symptomatic intracranial atherosclerosis. Cerebrovasc Dis 2012;33:517-524.

14 Hetzel A, Guschlbauer B, Reinhard M: Time delay as a parameter for cerebrovascular reactivity in patients with severe carotid stenosis. Cerebrovasc Dis 2003;16:14-20.

15 Webster MW, Makaroun MS, Steed DL, Smith HA, Johnson DW, Yonas H: Compromised cerebral blood flow reactivity is a predictor of stroke in patients with symptomatic carotid artery occlusive disease. J Vasc Surg 1995;21:338-345.

16 Yonas H, Smith HA, Durham SR, Pentheny SL, Johnson DW: Increased stroke risk predicted by compromised cerebral blood flow reactivity. J Neurosurg 1993;79:483-489.

17 Rogg J, Rutigliano M, Yonas H, Johnson DW, Pentheny S, Latchaw RE: The acetazolamide challenge: imaging techniques designed to evaluate cerebral blood flow reserve. AJR Am J Roentgenol 1989;153:605-612.

18 Ozgur HT, Kent Walsh T, Masaryk A, Seeger JF, Williams W, Krupinski E, Melgar M, Labadie E: Correlation of cerebrovascular reserve as measured by acetazolamide-challenged SPECT with angiographic flow patterns and intra- or extracranial arterial stenosis. AJNR Am J Neuroradiol 2001;22:928-936. 